\title{
An Unexpected Complication by Misplacement of an External Ventricular Drain; Transient Oculomotor Palsy Due to Direct Compression by a Drain-A Case Report
}

\author{
Hidenori Anami, Yasuo Aihara*, Yuki Takano, Kentaro Chiba, Seiichiro Eguchi, \\ Kohji Yamaguchi, Yoshikazu Okada, Takakazu Kawamata \\ Department of Neurosurgery, Tokyo Women's Medical University, Tokyo, Japan \\ Email: "yaihara@nij.twmu.ac.jp
}

Received 2 May 2016; accepted 31 May 2016; published 3 June 2016

Copyright (C) 2016 by authors and Scientific Research Publishing Inc.

This work is licensed under the Creative Commons Attribution International License (CC BY).

http://creativecommons.org/licenses/by/4.0/

(c) (i) Open Access

\section{Abstract}

Background: Insertion of an external ventricular drain (EVD) is an important neurosurgical technique. There is a minor risk of misplacement, and complications including infections or hemorrhages have been reported. Transient neurological complications are, however, very rare, especially when EVD is placed under endoscope assistance. We report a case of unilateral transient oculomotor palsy after an EVD procedure. Case presentation: An 11-year-old boy with past medical history of moyamoya disease suffered from intraventricular hemorrhage and acute hydrocephalus. Insertion of EVD and hematoma removal was performed with endoscope assistance. After the surgery, transient oculomotor palsy occurred by the direct compression of the brain stem by the drainage tube. Conclusion: Transient unilateral oculomotor palsy due to the direct compression of the midbrain by an EVD tube is a very rare, but possible complication, even under endoscopic assistance. Attention through the procedure is required until skin closure to avoid tube dislocation.

\section{Keywords}

External Ventricular Drain, Malplacement, Oculomotor Palsy

\footnotetext{
"Corresponding author.

How to cite this paper: Anami, H., Aihara, Y., Takano, Y., Chiba, K., Eguchi, S., Yamaguchi, K., Okada, Y. and Kawamata, T. (2016) An Unexpected Complication by Misplacement of an External Ventricular Drain; Transient Oculomotor Palsy Due to Direct Compression by a Drain-A Case Report. Journal of Behavioral and Brain Science, 6, 249-253. http://dx.doi.org/10.4236/jbbs.2016.66025
} 


\section{Introduction}

The insertion of an external ventricular drain (EVD) is one of the basic neurosurgical techniques widely used in the management of hydrocephalus or increased intracranial pressure. There have been multiple reports and reviews about complications of EVD insertion before. Almost all the reports are related to infections or hemorrhages, and the rate of misplacement is ranging from $12.3 \%$ to $60.1 \%$ [1]-[5]. Ngo et al. reported the rate of malplacement in pediatric cases at approximately 3\% [6]. Transient neurological complications are apparently very rare and to our best knowledge, there is only one case report of transient coma due to the direct compression of the brain stem by a drain [7]. We report a case of transient unilateral oculomotor palsy due to direct midbrain compression by an EVD.

\section{Case Presentation}

An 11-year-old boy with past medical history of moyamoya disease underwent bilateral direct STA-MCA double anastomoses in 2012. After 2 years, he was transferred to our hospital because of a generalized seizure and impairment of consciousness. His consciousness level on arrival was Glasgow coma scale (GCS) E1V1M3, and his pupils were 3.0 in diameter bilaterally with brisk light reflex. Head CT scan showed diffuse intraventricular hemorrhage (IVH) and acute hydrocephalus (Figure 1).

An urgent insertion of ventricular drain and hematoma evacuation were performed. A burr hole was made in the mid-orbital line over the left coronal suture, and a transparent sheath (Neuroport; Olympus Corp., Tokyo) was inserted into the anterior horn of the left lateral ventricle. Removal of intraventricular hematoma and septostomy were performed using an endoscope (OLYMPUS VEF TYPE V) under general anesthesia. An external ventricular drain (SILASCON E-4L-6, KANEKA MEDICAL PRODUCTS, Japan) was placed in the $3^{\text {rd }}$ ventricle to prevent its obstruction by residual hematoma. The drain was fixed to the skin before the wound was closed with 3-0 Polysorb and staplers. Just after the operation, when we removed the drape, his pupils showed anisocoria (Right $5.0 \mathrm{~mm} /$ Left $2.0 \mathrm{~mm}$ ) and there was no light reflex on the right side. Postoperative CT revealed no new hematoma or other abnormal lesion, but the tip of the drain was located in the interpeduncular cistern compressing the midbrain (Figure 2). We immediately pulled out the drain approximately $2 \mathrm{~cm}$, and his right pupil shrank to $3.5 \mathrm{~mm}$ in diameter with recovered light reflex on the right side. A repeated CT showed the tube not compressing the brain stem. Oculomotor palsy recovered 2 months after surgery (Figure 3).

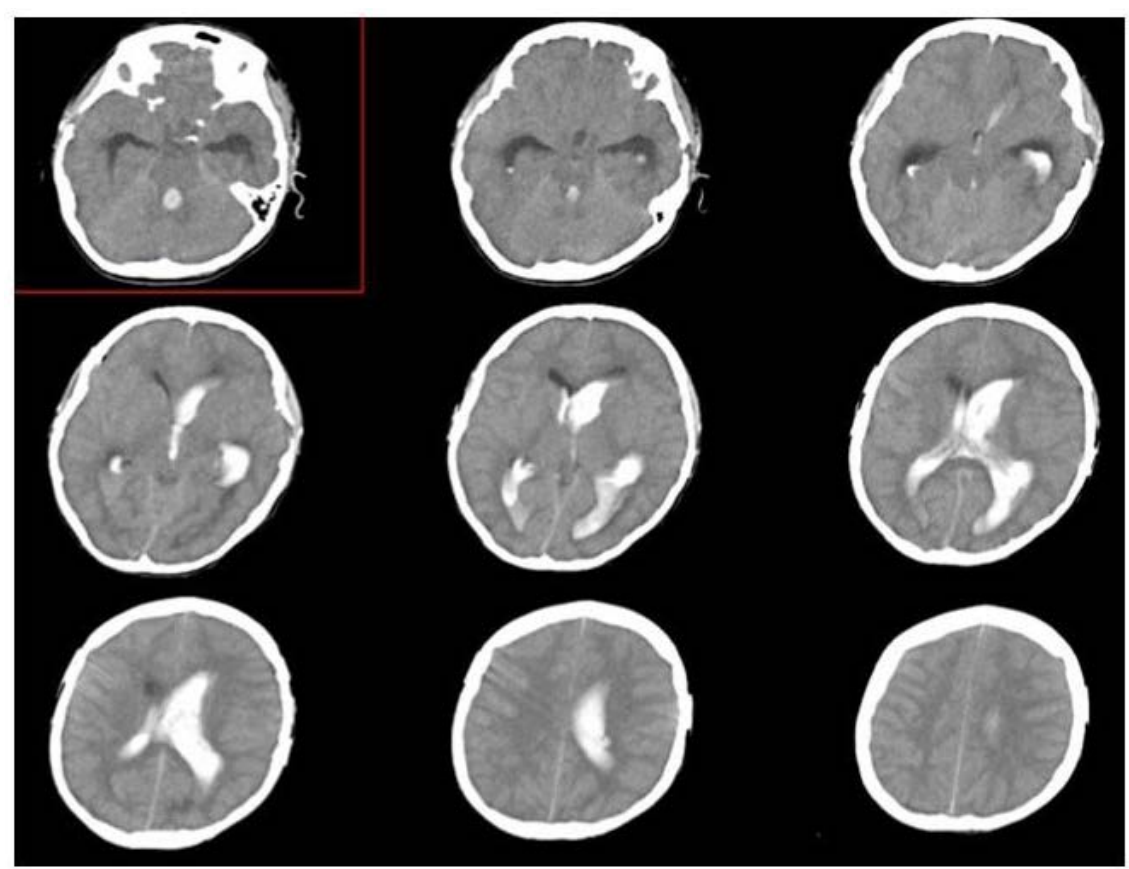

Figure 1. Head CT on admission shows diffuse intraventricular hemorrhage (IVH) and acute obstructive hydrocephalus. 


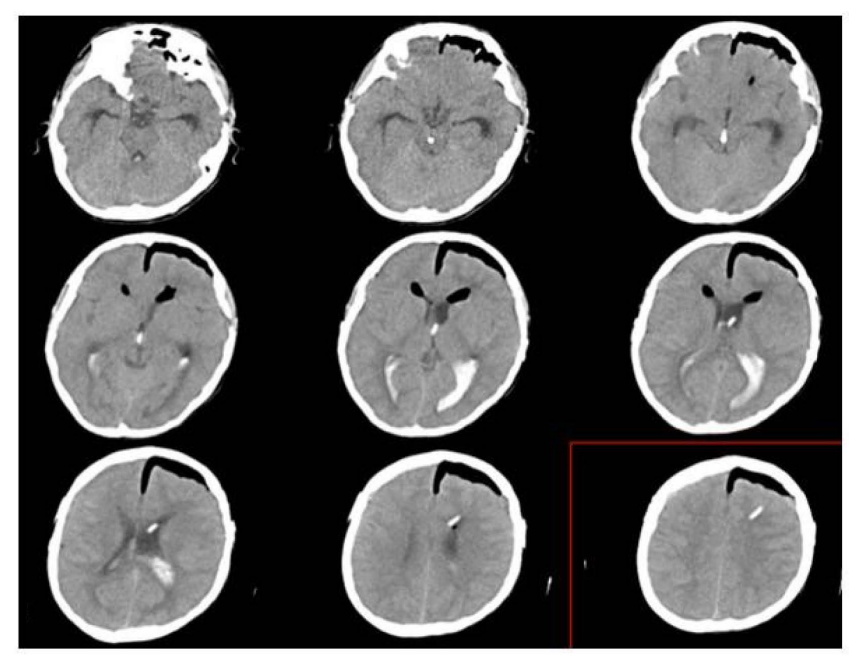

(a)

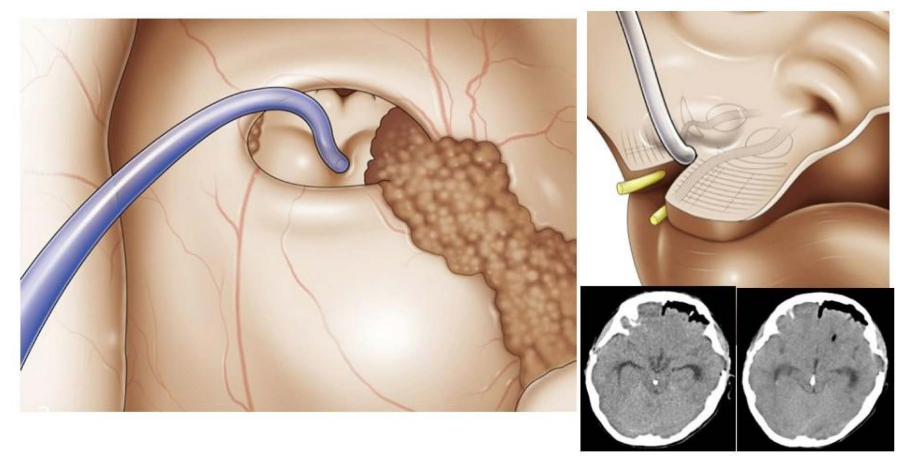

(b)

Figure 2. (a) Post operative CT shows that most IVH was removed and the tip of the drain was located in the interpeduncular cistern; (b) Schematic image shows the relation between brain stem and the drain.

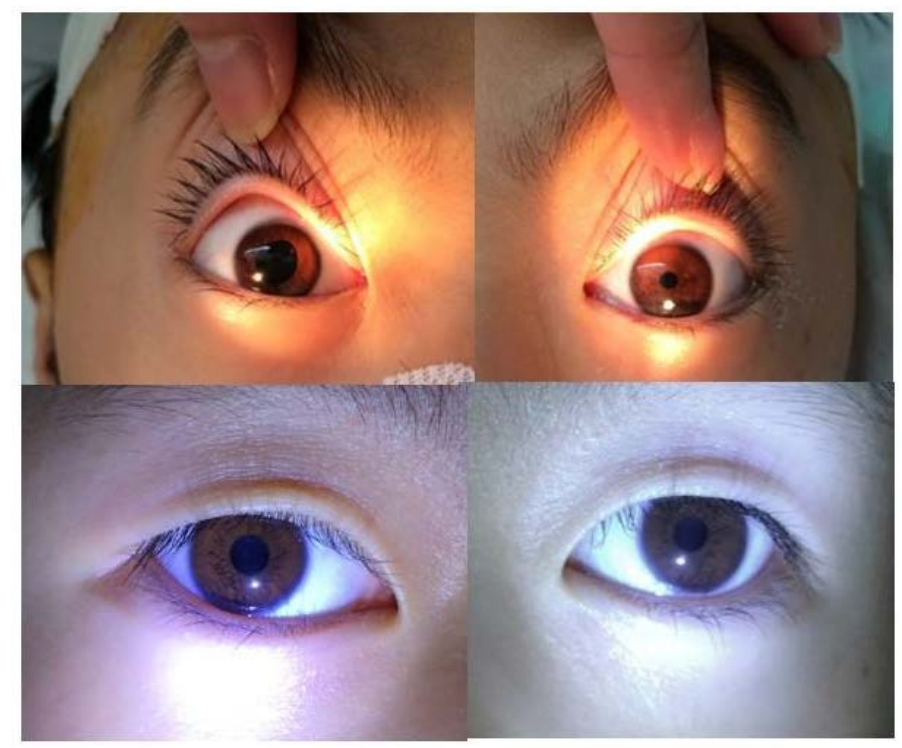

Figure 3. Photograph of pupils. Upper row: just after operation, right pupil was dilated without light reflex; Lower row: 2 months after operation, right oculomotor palsy recovered. 


\section{Discussion}

There are many case reports or literature reviews on complications related to the insertion of EVD. Almost all complications are related to infection or hemorrhage [5]. Misplacement of EVD can occur at times, but it is very rare to be associated with transient neurological complications [1]-[4]. There is only one report on transient disturbance of consiousness. Chai et al. had reported a case of transient coma due to brain stem compression by a drain [7]. In their case, the drain was inserted by free hand technique, and the tip of the drain penetrated into the 4th ventricle through the aqueduct. The tube was immediately pulled out and adjusted to release the brain stem compression and patient's consciousness level improved to GCS14/15 (E3V5M6) one hour after that.

In our case, the drain was placed in the third ventricle with endoscope assistance to avoid its obstruction by residual hematoma. Postoperative CT showed the tube to have moved slightly deeper with the tip of the tube penetrating into the interpeduncular cistern. The drain was inserted from the left lateral ventricle, and it compressed the opposite (right) side of midbrain and/or right oculomotor nerve itself.

The position of a ventricular drainage tube is important for its effectiveness. We placed the tip of the drain in the 3rd ventricle because we were afraid that a residual clot in the contralateral ventricle might obstruct the 3rd ventricle CSF flow. By placing the drain under direct observation using endoscope, we expected to guarantee its proper position.

We suspect that the tube might have been dislocated when we were closing the skin.

To avoid this, an important point in appropriate surgical technique is the direction of exiting of the tube at skin level. In our surgical procedure, the drain exited at a right angle to the skin incision direction. To reduce the risk of drain displacement, it might be better to pull out the tube parallel to the skin incision (Figure 4). We also should keep in mind the exact required length of the inserted drain and the possibility of tube displacement until we close the skin. If the tip of the drain is close to vital structures, the drain should be pulled out parallel to the skin incision for prevention of tube displacement.

\section{Conclusion}

EVD insertion is an important neurosurgical technique. There is some risk of misplacement, and complications including infections or hemorrhages have been reported before. However, it is very rare to observe transient neurological complications. We report a case of transient unilateral oculomotor palsy due to the direct compression of midbrain by an EVD tube. This kind of oculomotor palsy was reversible when the compression of brain stem was removed immediately. It is necessary to keep in mind that if a EVD tube is displaced, it might cause unexpected complications.

\section{Acknowledgements}

We thank Dr. Kostadin Karagiozov for his advice and manuscript review and gratefully acknowledge the radiological technologists, nurses, and staff of Departments of Neurosurgery, Tokyo Women's Medical University in preparing this paper.

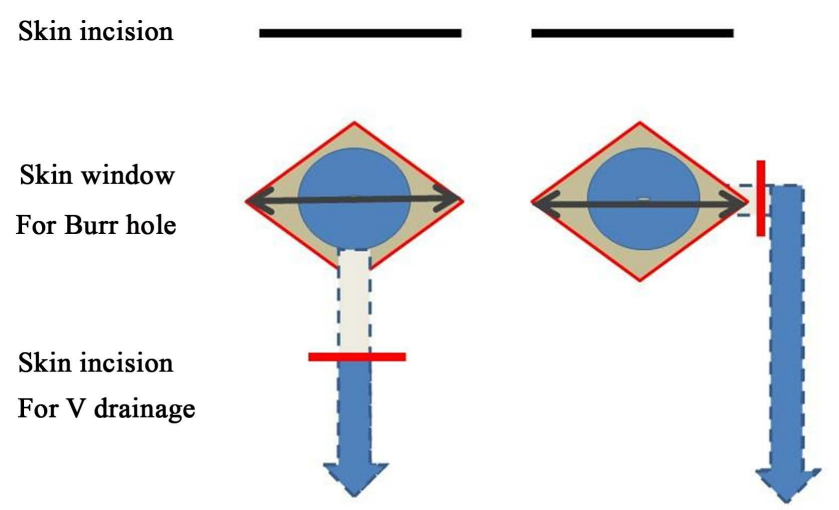

Figure 4. The relation between the drain and skin incision. The direction of exiting of the tube at skin level is important. 


\section{Funding}

This study was supported by research funds of Department of Neurosurgery, Tokyo Women's Medical University.

\section{Conflict of Interest}

The authors have no personal financial or institutional interest in any of the materials or devices described in this article.

\section{References}

[1] Kakarla, U.K., Kim, L.J., Chang, S.W., Theodore, N. and Spetzler, R.F. (2008) Safety and Accuracy of Bedside External Ventricular Drain Placement. Operative Neurosurgery, 63, ONS162-ONS166, Discussion ONS166-ONS167. http://dx.doi.org/10.1227/01.neu.0000312390.83127.7f

[2] Park, Y.G., Woo, H.J., Kim, E. and Park, J. (2011) Accuracy and Safety of Bedside External Ventricular Drain Placement at Two Different Cranial Sites: Kocher's Point versus Forehead. Journal of Korean Neurosurgical Society, 50, 317-321. http://dx.doi.org/10.3340/jkns.2011.50.4.317

[3] Saladino, A., White, J.B., Wijdicks, E.F. and Lanzino, G. (2009) Malplacement of Ventricular Catheters by Neurosurgeons: A Single Institution Experience. Neurocritical Care, 10, 248-252. http://dx.doi.org/10.1007/s12028-008-9154-Z

[4] Toma, A.K., Camp, S., Watkins, L.D., Grieve, J. and Kitchen, N.D. (2009) External Ventricular Drain Insertion Accuracy: Is There a Need for Change in Practice? Neurosurgery, 65, 1197-1200, Discussion 1200-1191.

[5] Woernle, C.M., Burkhardt, J.K., Bellut, D., Krayenbuehl, N. and Bertalanffy, H. (2011) Do Iatrogenic Factors Bias the Placement of External Ventricular Catheters? A Single Institute Experience and Review of the Literature.

[6] Ngo, Q.N., Ranger, A., Singh, R.N., Kornecki, A., Seabrook, J.A. and Fraser, D.D. (2009) External Ventricular Drains in Pediatric Patients. Pediatric Critical Care Medicine, 10, 346-351. http://dx.doi.org/10.1097/pcc.0b013e3181a320cd

[7] Chai, F.Y., Farizal, F. and Jegan, T. (2013) Coma due to Malplaced External Ventricular Drain. Turkish Neurosurgery, 23, 561-563. 